\title{
The role of blood vessels and lungs in the dissemination of Naegleria fowleri following intranasal inoculation in mice
}

\author{
Kirby L. Jarolim ${ }^{1}$, Jeffrey K. McCosh ${ }^{1}$, and Marsha J. Howard ${ }^{2}$ \\ ${ }^{1}$ Department of Anatomy and Cell Biology, Oklahoma State University Center for Health Sciences, 1111 West $17^{\text {th }}$ Street, Tulsa, \\ OK 74107, USA; \\ ${ }^{2}$ Office of Basic Science, Oklahoma State University Center for Health Sciences, 1111 West $17^{\text {th }}$ Street, Tulsa, OK 74107, USA
}

Key words: Naegleria fowleri, primary amoebic meningoencephalitis, mouse, intranasal inoculation, blood culture, lungs

\begin{abstract}
Primary amoebic meningoencephalitis (PAM) was induced in mice by intranasal inoculation of Naegleria fowleri (Singh et Das, 1970) to study the role of the blood vessels and lungs in the early and later stages in this disease. Upon culturing blood and lung tissue obtained at 24-, 36-, 48-, 72-, 96-, and 120-hour time periods, it was found that amoebae grew only from blood and lung tissue obtained at the 96 and 120 hour time periods. Paraffin sections of the head revealed small foci of acute inflammation and amoebae within the olfactory bulb of the central nervous system (CNS) at 24 hours. Amoebae were not observed within blood vessels of the CNS until 96 and 120 hours. Also, amoebae were observed within the connective tissue surrounding blood vessels and sutures of the skull, bone marrow, and venous sinusoids between the skull bone tables at 96 and 120 hours. No amoebae or acute inflammatory reactions were observed in the lung sections from any time period and indirect immunofluorescence microscopy was negative for $N$. fowleri. This study provides evidence that neither blood vessels nor lungs provide routes for $N$. fowleri to the CNS during the early stages of PAM and that amoebae enter veins of the CNS and bone marrow during later stages of the disease.
\end{abstract}

Naegleria fowleri (Singh et Das, 1970) is a freeliving amoeboflagellate that has been identified as the etiological agent of primary amoebic meningoencephalitis (PAM) in humans when inspired into the nasal cavity (Martinez 1985, John 1993). A condition similar to human PAM can be experimentally produced in mice when inoculated intranasally with $N$. fowleri (Duma 1972, Martinez et al. 1973b). The primary pathway taken by $N$. fowleri from the nasal mucosa to the central nervous system (CNS) during the initial stages of PAM has been elucidated (Jarolim et al. 2000). The possible involvement of the blood vessels and lungs in early stage dissemination of this rapidly fatal disease remains a question. Martinez et al. (1973a) reported that they occasionally found amoebae in blood vessels of moribund mice following intranasal inoculation. They stated that the blood vessels appear to play no important role in infecting the CNS, as organisms could not be found in other parts of the body.

To elucidate the role of blood vessels and the lungs in the progression of PAM, mice were inoculated intranasally with $N$. fowleri and killed at various postinoculation time intervals. Blood and lung tissue samples were cultured to detect the presence of amoebae. Histological sections of the olfactory bulb and nasal mucosa adjacent to the cribriform plate were analysed through the time periods to identify amoebae entering or within the blood vessels. Also, lung sections from the same time groups were studied with light and fluorescence microscopy for the presence of amoebae in the pulmonary vasculature and the intrapulmonary airways.

\section{MATERIALS AND METHODS}

Amoebae and cultivation. Amoebae used in this study were $N$. fowleri (Lee-mp), passaged in mice to retain maximum virulence. Amoebae were grown axenically in Mix amoeba medium (John 1993). Early stationary-growth-phase amoebae were harvested by centrifugation $(1,200 \mathrm{~g}, 10 \mathrm{~min}$, $20^{\circ} \mathrm{C}$ ), washed three times in Page's amoeba saline (Page 1988), and suspended in Page's saline. Cell counts were made with a Coulter counter (model $\mathrm{Z}_{\mathrm{BI}}$ Coulter Electronics, Hialeah, Florida) using settings previously described (John and John 1989, Jarolim et al. 2000).

Mice and inoculation. Male, 21-day-old, CD-1 outbred mice (Charles River Laboratories, Wilmington, Massachusetts) were used in all experiments. All mice were kept in isolation for 2 or 3 days before experimentation. The mice were given free access to water and feed (Purina Lab Chow, Ralston Purina Corp., St. Louis, Missouri).

Mice were lightly anaesthetised with Metofane (PitmanMoore, Washington Crossing, New Jersey) and inoculated by intranasal instillation of a $10 \mu \mathrm{l}$ suspension of amoebae in Page's amoeba saline. A single $10 \mu \mathrm{l}$ drop containing $1 \times 10^{6}$ amoebae was introduced into the left nostril using an Eppendorf pipette (Brinkman Instruments, Westbury, New York). The right nostril was not inoculated. Mice were separated into groups of 3 and killed at time periods of 24, 32, $48,72,96$, and 120 hours postinoculation. No mice died before the end of the time periods studied (Jarolim et al. 2000).

Blood sample retrieval and culture. Mice were anaesthetised with a single intraperitoneal injection of sodium pentobarbital $(50 \mathrm{mg} / \mathrm{kg} \quad$ b.w.). Following a midline 
thoracotomy, the animals were exsanguinated through the ventricles of the heart using a 3-cc heparinised syringe with a 25 -gauge hypodermic needle. The blood samples were immediately transferred into media in $75-\mathrm{cm}^{2}$ polystyrene tissue culture flasks (Corning Glass Works, Corning, New York). The medium consisted of Mix amoeba medium supplemented with $200 \mu \mathrm{g}$ streptomycin/ml and $200 \mathrm{U}$ penicillin/ml (Sigma). Mix amoeba medium is an equal mixture of Balamuth (Balamuth 1964) and Nelson (Nelson and Jones 1970) media that is $0.55 \%$ liver digest, $0.50 \%$ proteose peptone, $0.25 \%$ yeast extract, and $0.30 \%$ glucose in Page amoeba saline $\left(0.12 \mathrm{~g} \mathrm{NaCl}, 0.004 \mathrm{~g} \mathrm{MgSO}_{4} \cdot 7 \mathrm{H}_{2} \mathrm{O}\right.$, $0.004 \mathrm{~g} \mathrm{CaCl}_{2} \cdot 2 \mathrm{H}_{2} \mathrm{O}, 0.142 \mathrm{~g} \mathrm{Na}_{2} \mathrm{HPO}_{4}$, and $0.136 \mathrm{~g} \mathrm{KH}_{2} \mathrm{PO}_{4}$ per litre of distilled water; Page 1988) supplemented with $4 \%$ bovine calf serum and $1 \mu \mathrm{g}$ hemin $/ \mathrm{ml}$. The flasks were incubated at $37^{\circ} \mathrm{C}$ and were examined periodically for 3 weeks using inverted bright-field microscopy for evidence of amoebae.

Tissue sample retrieval and processing. Following exsanguinations, the left lungs were removed, minced with Teflon coated razor blades, and the pieces transferred to 25$\mathrm{cm}^{2}$ polystyrene tissue culture flasks (Corning). The flasks contained the same medium as described above and were incubated at the same temperature. The flasks were examined periodically for 3 weeks by inverted bright-field microscopy for evidence of amoebae.

The right lungs were sectioned into apical, middle, and basal segments for light microscopy. The lung segments were fixed in $10 \%$ neutral buffered formalin (NBF), dehydrated, and embedded in paraffin in the usual manner. Tissue sections were cut at $5 \mu \mathrm{m}$ and stained with a combination of fast green and cresyl violet acetate (FC) for light microscopy.

The mice were decapitated, skin and musculature removed from the heads, caudal aspect of the skull opened, and dropfixed for 72 hours in NBF. Following fixation, the heads were decalcified in a solution of trisodium citrate and formic acid for 48 hours (Emanuele 1992). Next, the decalcified heads were divided in the mid-sagittal plane with Teflon coated razor blades. The cribriform plate was identified and a rectangular block of tissue with the cribriform plate at the centre was removed from each half of the head. Care was given to make the cuts parallel to the angle of the cribriform plate. The blocks of tissue included the olfactory bulb and a portion of the nasal cavity on respective sides of the cribriform plate. The tissue blocks were equilibrated in $5 \%$ trisodium citrate, washed in water, then dehydrated and embedded in paraffin in the usual manner. Serial sections were cut at $5 \mu \mathrm{m}$ for light microscopy and the sections were stained with FC.

Indirect immunofluorescence study. Representative lung tissue sections from the 96- and 120-hour groups were incubated with a 1:128 dilution of polyclonal antisera, raised in rabbits against $N$. fowleri, for $30 \mathrm{~min}$ at $37^{\circ} \mathrm{C}$ (John et al. 1998). All unbound primary antibody was removed by washing the sections with phosphate buffered saline. Next, the sections were incubated with a 1:150 dilution of goat antirabbit IgG conjugated with Alexa 488 fluorophore (Molecular Probes, Eugene, Oregon) for $30 \mathrm{~min}$ at $37^{\circ} \mathrm{C}$. Mouse olfactory bulb tissue sections containing $N$. fowleri served as an external positive control. Lung tissue from a normal mouse was treated as above and served as the negative control.

All light and fluorescence microscopy observations and photomicrography were performed with a Zeiss photomicroscope III. Kodak Technical Pan film was used for light photomicrography and Kodak TMAX 400 film with a XF56 filter set (Omega Optical, Battleboro, Vermont) was used for immunofluorescence (IF) photomicrography.

\section{RESULTS}

\section{Blood cultures}

Blood cultures from mice in the 24-, 32-, 48-, and 72hour postinoculation groups remained negative for amoebae throughout the 3-week observation period. Cultures from the 96- and 120-hour groups became positive for amoebae on day 12 of the observation period.

\section{Lung cultures}

Lung cultures from mice in the 24-, 32-, 48-, and 72hour postinoculation groups remained negative for amoebae throughout the 3 -week observation period. The cultures from the 96- and 120-hour groups became positive for amoebae on day 8 of the observation period.

\section{Light microscopy study of the cribriform plate region}

Amoebae and acute inflammatory foci were observed in the olfactory nerves and CNS as early as 24 hours postinoculation. The number of amoebae and amount of inflammatory response increased with time. These observations have been previously reported (Jarolim et al. 2000). As the number of amoebae and inflammatory reaction increased, the olfactory bulb and adjacent areas of the brain became necrotic. In the 96- and 120-hour groups, amoebae could occasionally be seen entering dilated thin-walled blood vessels (Fig. 1), within the connective tissue surrounding the blood vessels (Fig. 2) and sutures of the skull, and within the bone marrow and its venous sinusoids between the bone tables of the skull (Figs. 3, 4). Larger arterial blood vessels appeared to remain intact through all time periods studied.

\section{Light and immunofluorescence microscopy study of lung tissue}

Amoebae were not observed in FC (Fig. 5) or IF (Fig. 6) treated lung sections of the 96- and 120-hour groups. This included the blood vessels, interstitial areas, and intrapulmonary airways. The control olfactory bulb tissue sections used for the IF procedure were from the 120-hour group and were positive and specific for $N$. fowleri (Fig. 7). The negative control lung tissue and sections from all time groups demonstrated only background non-specific fluorescence. 


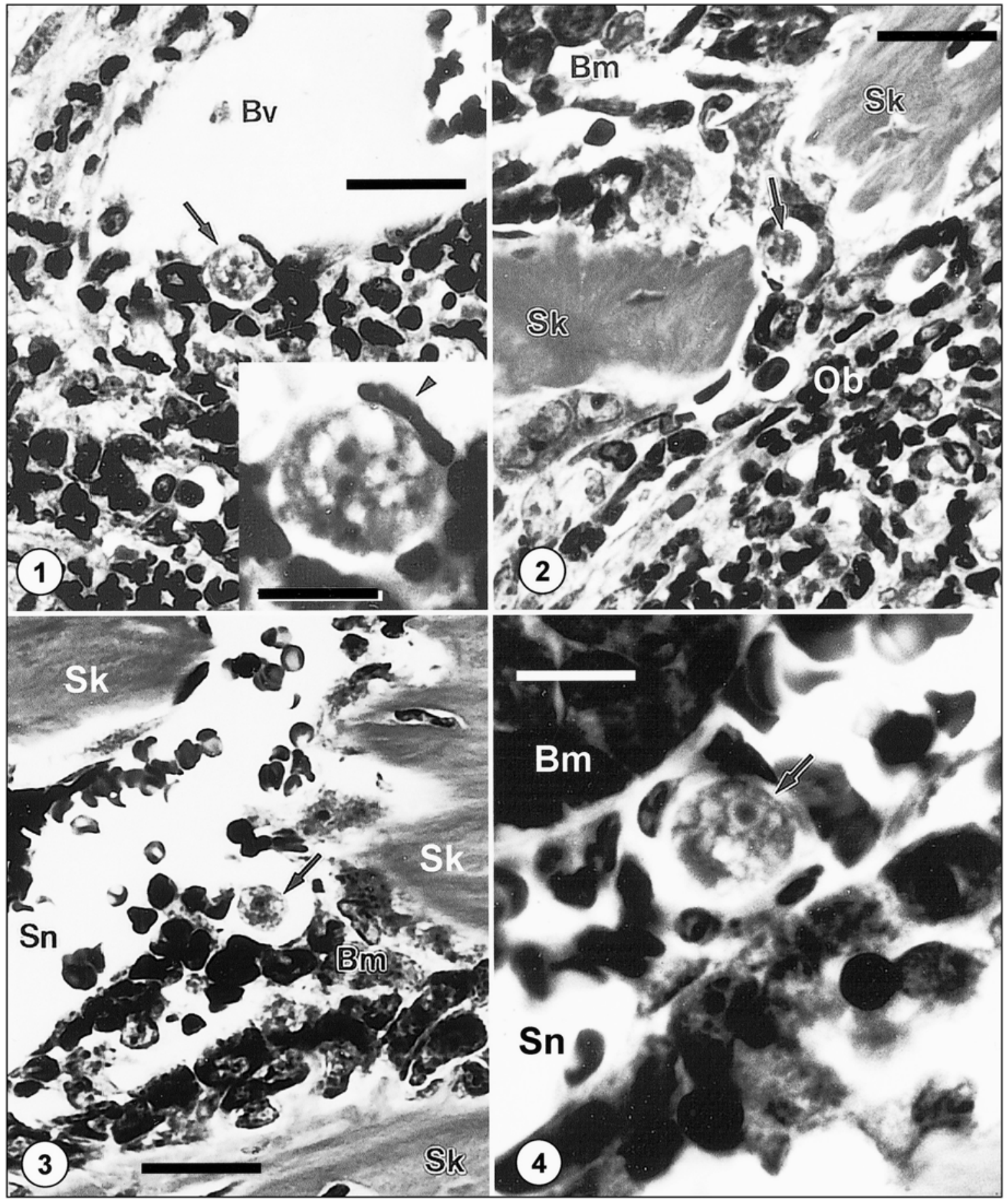

Figs. 1-4. Photomicrographs of Naegleria fowleri within the olfactory bulb and bone marrow of mice in terminal stages of primary amoebic meningoencephalitis. Fig. 1. Intermediate magnification of the olfactory bulb demonstrates extensive acute inflammation and necrosis in a mouse from the 96-hour group. The arrow indicates an amoeba entering a dilated thin-walled vein (Bv). The inset is a higher magnification of the same amoeba. Note the endothelial cell nucleus (arrowhead) pushed into the lumen of the vein by the highly vacuolated amoeba. Fig. 2. An amoeba (arrow) moving through the cellular connective tissue surrounding a blood vessel penetrating the skull of a mouse from the 96-hour group. The clear halo surrounding the amoeba is due to fixation shrinkage artifact. $\mathrm{Bm}$ - bone marrow between skull bone tables; Ob - olfactory bulb; Sk - skull bone table. Fig. 3. Section from a mouse in the 96-hour group demonstrating an amoeba (arrow) approaching a venous sinusoid (Sn) from the bone marrow (Bm). Sk - skull bone table. Fig. 4. High-power magnification of a section from a mouse in the 96-hour group. Note the amoeba (arrow) with a prominent karyosome surrounded by white blood cells within a venous sinusoid ( $\mathrm{Sn})$. Bm - bone marrow. Scale bars: Fig. $1=20 \mu \mathrm{m}$, inset $=8 \mu \mathrm{m}$; Figs. $2,3=20 \mu \mathrm{m}$; Fig. $4=8 \mu \mathrm{m}$. 



Figs. 5-8. Photomicrographs of lung, bone marrow, and nasal mucosa of mice inoculated with Naegleria fowleri. Fig. 5. Lung section from a mouse in the 96-hour group. The lung appears normal. Note the lack of inflammation within the intrapulmonary bronchus (Ib), alveoli (Al), and blood vessel (arrow). Fig. 6. Lung section from a mouse in the 120-hour group. Note the lack of specific fluorescence for amoebae in the alveoli (Al) and intrapulmonary bronchi (Ib). The dull non-specific fluorescence in tissue between the alveoli is the autofluorescence of red blood cells within capillaries. Fig. 7. Section of immunofluorescence control tissue from the olfactory region of an infected mouse in the 120-hour group. Note the specific fluorescence of amoebae within the olfactory bulb $(\mathrm{Ob})$ and the bone marrow $(\mathrm{Bm})$ between the bone tables of the overlying skull (Sk). Fig. 8. The mucosal surface within the nasal cavity $(\mathrm{Nc})$ of a mouse from the 48-hour group demonstrates a thick inflammatory exudate containing numerous acute inflammatory cells (Ex). An amoeba is identified by the arrow. Ep - olfactory epithelium; Sp submucosal nerve plexus; ${ }^{*}$ - cribriform plate. Scale bars: Fig. $5=35 \mu \mathrm{m}$; Figs. $6,7=50 \mu \mathrm{m}$; Fig. $8=70 \mu \mathrm{m}$. 


\section{DISCUSSION}

Intranasal inoculation of mice with Naegleria fowleri amoebae results in a rapidly fulminating and fatal disease similar to that seen in human PAM (Martinez et al. 1973a). The route the amoebae take to enter the CNS has been demonstrated from the nasal mucosa, within the olfactory fila penetrating the cribriform plate of the ethmoid bone, to the olfactory bulb of the brain (Martinez et al. 1973a, Jarolim et al. 2000). One wonders whether there is involvement of the blood vessels and lungs in the dissemination of the disease following inoculation of the nasal cavity. In the present study, the progression of PAM was followed from the early stages to the point where the mice were moribund (120 hours). Since the blood samples remained negative from 24 through 72 hours postinoculation, it appears that blood vessels are not involved as an additional route for the amoebae to enter the CNS during these time periods. However, in the later stages of the disease (96 and 120 hours) the blood samples became positive for amoebae. When correlating this finding with that observed in the histological sections of the cribriform plate area of the same time periods, it becomes evident that this may be due to the advanced tissue destruction that occurs by the 96-hour time period. The thinnerwalled blood vessels (veins) of the CNS were dilated and amoebae were occasionally observed between the endothelial cells (Fig. 1). The thicker-walled arterial vessels appear to remain intact throughout all time periods. Other significant observations were the presence of amoebae within the connective tissue surrounding blood vessels of the skull, within the sutures of the skull, and within the bone marrow and venous sinusoids between the bone tables of the skull. These previously unreported observations may be additional routes by which $N$. fowleri enter the blood stream (Figs. 2-4) during the later stages of PAM. Martinez et al. (1973a) stated that the blood vessels play no important role in the infection of the CNS. Their finding is confirmed by this study and it is concluded that the amoebae enter compromised dilated veins in the olfactory and adjacent brain areas after the amoebae are already established in the CNS.

The cultures of the lung were initially negative but became positive during the same time periods as the blood cultures (96 and 120 hours). However, histological sections and immunofluorescence studies did not demonstrate any pathology or presence of amoebae in any aspect of the lung structure. This curious finding may be due to the small number of amoebae within the blood stream that moved from necrotic areas of the brain and the bone marrow sinusoids to the pulmonary circulation within the time periods studied. From these observations it is apparent that the amoebae do not enter the lungs through the intrapulmonary airways from the nasal cavities since no acute inflammatory reaction or amoebae were observed in any of the bronchi, bronchioles, or alveolar spaces. If this were the case, one would expect to see acute inflammation within the intrapulmonary airways like that seen in the mucosa of the nasal cavities in the earlier stages of PAM (Fig. 8). Since the lung capillary beds are extensive, it is likely that there may be isolated amoebae circulating in the pulmonary vasculature and, hence, the reason for the positive lung cultures. Since no amoebae were detected in the FC-stained sections, the more sensitive immunofluorescence procedure specific for $N$. fowleri was employed. Since only a few sections from the later time periods were used for immunofluorescence, there is a distinct possibility that amoebae in the pulmonary blood vessels could have been missed.

From this study, it is concluded that $N$. fowleri does not use blood vessels or the lungs as routes to the CNS during the early stages of PAM. However, amoebae enter the blood stream of the CNS and bone marrow very late in the disease process when the mice are moribund, tissue destruction is advanced, and the thinwalled veins of the CNS are compromised. Also, the FC stain developed by one of the authors (JKM) proved to be a rapid and efficient histological stain for differentiating $N$. fowleri from surrounding tissues of the CNS.

Acknowledgements. We are indebted to David T. John for supplying the $N$. fowleri cultures used in this study and for his critical review of the manuscript. The authors wish to thank Oza M. McClain for help with the immunofluorescence technique and Gloria Mercier for secretarial support. This investigation was supported with funds provided by the Office of Research, Oklahoma State University Center for Health Sciences.

\section{REFERENCES}

BALAMUTH W. 1964: Nutritional studies on axenic cultures of Naegleria gruberi. J. Protozool. 11 (Suppl.): 19-20.

DUMA R.J. 1972: Primary amoebic meningoencephalitis. CRC Critical Reviews in Clinical Laboratory Sciences 3: 162-192.

EMANUELE P.V. 1992: Ocular histotechnology. In: E. Prophet, B. Mills, J. Arrington and L. Sobin (Eds.), Laboratory Methods in Histotechnology. American Registry of Pathology, Washington, D. C., pp. 109-110.
JAROLIM K.L., McCOSH J.K., HOWARD M.J., JOHN D.T. 2000: A light microscopy study of the migration of Naegleria fowleri from the nasal submucosa to the central nervous system during the early stage of primary amoebic meningoencephalitis in mice. J. Parasitol. 86: 50-55.

JOHN D.T. 1993: Opportunistically pathogenic free-living amoebae. In: J. Kreier and J. Baker (Eds.), Parasitic Protozoa, Second Edition, Vol. 3. Academic Press, San Diego, California, pp. 143-246. 
JOHN D.T., HOWARD M.J., WATSON K.R. 1998: Identification of environmental isolates of pathogenic Naegleria amoebae by indirect immunofluorescence. Proc. Okla. Acad. Sci. 78: 11-14.

JOHN D.T., JOHN R.A. 1989: Cytopathogenicity of Naegleria fowleri in mammalian cell cultures. Parasitol. Res. 76: 20-25.

MARTINEZ A.J. 1985: Free-living Amoebae: Natural History, Prevention, Diagnosis, Pathology, and Treatment of Disease. CRC Press, Boca Raton, Florida, 156 pp.

MARTINEZ A.J., DUMA R.J., NELSON E.C., MORETTA F.L. 1973a: Experimental Naegleria meningoencephalitis in mice. Penetration of the olfactory mucosal epithelium by Naegleria and pathologic changes produced: a light and electron microscope study. Lab. Invest. 29: 121-133.

Received 10 July 2001
MARTINEZ A.J., NELSON E.C., DUMA R.J. 1973b: Animal model: primary amoebic (Naegleria) meningoencephalitis in mice. Am. J. Pathol. 73: 545-548.

NELSON E.C., JONES M.M. 1970: Culture isolation of agents of primary amebic meningoencephalitis. Proc. $2^{\text {nd }}$ Int. Congr. Parasitol., Washington, D.C., 6-12 September 1970. Resume No. 450. J. Parasitol. 56 (Section II, Part I): 248.

PAGE F .C. 1988: A New Key to Freshwater and Soil Gymnamoebae. Freshwater Biological Association, Ambleside, Cumbria, UK, 122 pp.

SINGH B.N., DAS S.R. 1970: Studies on pathogenic and nonpathogenic small free-living amoebae and the bearing of nuclear division on the classification of the order Amoebida. Philos. Trans. R. Soc. Lond. B 259: 435-476.

Accepted 8 February 2002 